Advance Publication

\title{
Experimental Animals
}

Received: 2021.4.19

Accepted: 2021.7.7

J-STAGE Advance Published Date: 2021.8.9 
$1 \quad$ Management

2 Original paper

3

4 Development of incident severity classification for laboratory animals

5

6 INCIDENT SEVERITY CLASSIFICATION

7

8 Gaku Wagai $^{1)}$, Masao Togao ${ }^{1)}$, Jun Otsuka ${ }^{1)}$, Yuki Ohta-Takada ${ }^{1)}$, Shoichi Kado ${ }^{1)}$, Koji

9 Kawakami ${ }^{1)}$.

10 1) Yakult Central Institute, 5-11 Izumi, Kunitachi-shi, Tokyo, 186-8650, Japan

11

12 Corresponding Author

13 Gaku Wagai,

14 Address: Yakult Central Institute, 5-11 Izumi, Kunitachi-shi, Tokyo, 186-8650, Japan

15 E-mail address: gaku-wagai@yakult.co.jp

16 


\section{Abstract}

18 An incident reporting system (IRS) prevents possible adverse events by collecting and analyzing incidents that occur. However, few studies are available regarding IRSs in the laboratory animal field. This study aimed to develop an incident severity classification

21 for laboratory animals (ISCLA) to evaluate the usefulness of the IRS in laboratory animal facilities. Twenty-three incidents reported from March 2019 to February 2020 on our IRS were retrospectively reviewed. Three of the 23 incidents failed to obtain some experimental data. Two of these incidents were harmless to animals, but the other caused the animals moderate distress. In addition, two of the three incidents made animals unsuitable for experiments. Since the inconsistent impact of incidents on animals and experiments prevented the comparison of the severity of individual incidents, we developed the ISCLA. According to the ISCLA, the above three incidents were classified into Category $3 \mathrm{~b}$ and $4 \mathrm{a}$. The others were classified into Category $0(n=$ $5), 1(n=6), 2(n=3)$, and $3 \mathrm{a}(n=6)$ in ascending order of severity. No incident was

31 classified into Category $4 \mathrm{~b}$ and 5. Furthermore, incidents occurring in the animal 32 housing area were more severe than those occurring in the supporting area $(P=0.002)$. This study showed that incident occurrences had characteristics that were not visible

34 from individual incidents alone. Moreover, the ISCLA was considered useful when conducting the IRS and taking improvement measures in laboratory animal facilities.

\section{Key Words} incident reporting system, prevention, severity classification 
Introduction

Unexpected incidents occur at laboratory animal facilities. Recently, studies regarding adverse events that are unexpected incidents that lead to harm or result in a negative effect on the well-being of animals have been published [1,2]. These studies encourage institutions to learn from the adverse events and to take appropriate measures to prevent possible adverse events in the future $[1,2]$. On the other hand, although many adverse events are preventable, preventive and mitigating steps are not well-developed $[1]$.

At our laboratory animal facility, we conducted an incident reporting system (IRS) to collect unexpected incidents that might lead and that actually led to harm to animals and experiment to analyze the causes of the incidents and to take improvement measures. The IRS has been used in the human medical field as one of the common methods to detect a human error that causes harm to patients [3]. In recent years, the IRS has also been used in the veterinary medical field [4-6]. Although few studies are currently available on the IRS in the laboratory animal field, the IRS has the potential to contribute toward positive consequences on laboratory animal welfare [7]. When properly conducted, the IRS is effective in allowing people to learn from incidents [3]. In the human medical field, to effectively conduct the IRS, some analytical methods and tools, such as the system for classification of harm on the patient,

59 have been developed [8]. Standardization of classification is attempted to compare 60 incident occurrences among multiple facilities [9]. However, no analytical method or 61 tool has been developed for the laboratory animal field. In this study, the incidents reported on our IRS were retrospectively reviewed to analyze the characteristics of the occurrence of incidents in a laboratory animal facility. 
64 We then developed an incident severity classification for laboratory animals (ISCLA) and evaluated the appropriateness and usefulness of the classification.

\section{Materials and Methods}

\section{Setting}

In this study, 23 incident report forms reported on our IRS from March 2019 to February 2020 were retrospectively reviewed.

\section{Animals}

In this study, there was no direct interaction with animals. Therefore, this study was not reviewed by an ethical committee. However, every animal study undertaken in our institute was reviewed by the Institutional Animal Care and Use Committee of Yakult Central Institute and approved by the Director of Yakult Central Institute. In the laboratory animal facility, mice and rats were housed under the animal care and use program accredited by AAALAC International. Approximately $80 \%$ of them were specific pathogen-free animals housed in standard animal housing rooms. The others were germ-free and gnotobiotic animals housed in flexible film isolators.

\section{Incident reporting system}

Our IRS was conducted as follows: Personnel who were involved in or detected an incident took an initial action and reported the incident to the leader of animal caretakers. The incident and the initial action were reported in the daily meeting of animal caretakers. The reporter submitted an incident report, which included details of the incident, estimated factors that caused the incident, and improvement measures by using the incident report form described below. The laboratory animal manager and the attending veterinarian reviewed the report and advised on the improvement measures. The leader of animal caretakers finalized improvement measures. Finally, after the 
improvement measures were approved by the laboratory animal facility manager, they were implemented.

\section{Incident report form}

The incident report form consisted of the three following parts: the first report, review, and final report. The first report included the following: identification number of the report; title; reporter's name; name of personnel involved in or detecting the incident; date, time, and location of the incident or its detection; what the personnel did when the incident occurred or was detected; what happened, that is, a description of the event; problems and possible causes of the incident; and improvement measures. The review included the following: judgment of actual and potential impact of the incident on the animals, facility, and experiment by the attending veterinarian; advice from the attending veterinarian; and advice from the laboratory animal manager. The final report included the following: finalized improvement measures and date when the improvement measures were approved by the laboratory animal facility manager.

\section{Analysis of incidents}

The occurrence factors were classified into human factors, including slip, lapse, and mistake, and equipment factors, including equipment failure. The number of incidents by the occurrence factors was counted. Incidents associated with both human and equipment factors were counted as factors that are more relevant. The occupational groups of those who were mainly involved in the incidents associated with the human factor were classified into animal caretaker and investigator, and the number of occurrences by the groups was counted. The occupational groups of those who detected incidents were classified into animal caretaker, investigator, and manager, consisting of the attending veterinarian, leader of animal caretakers, laboratory animal managers, and laboratory animal facility manager, and the number of detections by the groups was 
114 counted. The number of occurrences of incidents by month and by day of the week was

115 counted. One incident whose occurrence date was unidentified was excluded from the

116 analysis of days of the week. In addition, the number of detections by day of the week

117 was also counted.

$118 \quad$ Analysis of incident severity

119 All incidents were analyzed according to the following criteria: whether the 120 incident actually affected the animal and experiment; continuity of actual harm to the

121 animal classified as none, transient, permanent, and death; severity of actual harm to the

122 animal classified as none, mild, moderate, and severe; severity of actual harm to the

123 experiment was classified as none, possibility, data missing, and not achieved; and

124 whether the animal was euthanized based on the discretion of a veterinarian.

Incident severity classification

All incidents were evaluated and classified into eight categories according to our incident severity classification. This classification system was developed with reference to the incident severity classification system, which is recommended by the National University Hospital Council of Japan [10].

The incident severity based on our severity classification was tabulated by the occurrence factors, i.e., the human and equipment factors. Similarly, the number of occurrences and incident severity by work locations were analyzed. The work locations where incidents occurred were classified into the supporting area, such as equipment preparation rooms and storage areas, and the animal housing area. The animal housing area was further divided into the general area, consisting of standard animal housing

137 rooms, and the isolator area, where animals, such as germ-free animals, were housed 138 with flexible film isolators. 


\section{Statistical analysis}

140 Evenness in the distributions of occurrences and the detections of incidents was

141 analyzed by an exact goodness-of-fit test on the xmulti function from the XNomial

142 package version 1.0.4 in $\mathrm{R}$ version 4.0.2. The comparison of severities of harm between

143 occurrence factors and between work locations was analyzed by a Mann-Whitney U

144 test on Bell Curve for Excel version 3.00 (Social Survey Research Information Co., Ltd.,

145 Japan). A $P$ value of $<0.05$ was considered statistically significant.

146

147 Results

$148 \quad$ Characteristics of reported incidents

149 Of the 23 reported incidents, $18(78.3 \%)$ were associated with the human factor 150 and five $(21.7 \%)$ to the equipment factor. Of the 18 human factor related incidents, 14 $151(77.8 \%)$ and $4(22.2 \%)$ were caused by errors of the animal caretakers and investigators, 152 respectively $(P=0.031)$. The majority of incidents $(n=19,82.6 \%)$ were detected by 153 animal caretakers. The other incidents were detected by investigators $(n=3,13.0 \%)$ and 154 by a manager $(n=1,4.3 \%)$.

155 Figure $1 \mathrm{~A}$ and $\mathrm{B}$ show the number of incidents by month, and the number of 156 occurrences and detections of incidents by day of the week, respectively. The incident 157 occurrences had no significant bias by month (Fig. $1 \mathrm{~A} ; P=0.696$ ). Occurrences of 158 incidents by day of the week were not even (Fig. 1B; $P=0.006$ ). Although no incident 159 occurred on the weekend, and a large number of incidents occurred on Monday and 160 Friday, the occurrences of incidents on weekdays had no significant bias by day of the 161 week $(P=0.134)$. The bias by day of the week in the number of detections of incidents 162 was not significant $(P=0.341)$. 
The impact of the 23 incidents on animals and experiments and the need for euthanasia of animals are summarized in Figure 2. Of the 23 incidents, five did not affect both animals and experiments, and six resulted in no harm on both of them. Of

167 the other 12 incidents, three resulted in transient and mild harm to the animals, which

168 required no treatment, but no harm to the experiments. Of the other nine incidents, six resulted in transient and moderate harm to the animals, which required simple treatment. In four of these six incidents, the experiments were not harmed. In the other two incidents, although the possibility of an effect on the experiments was not possible to

172 exclude, the primary purpose of the experiments had been achieved. Of the other three

173 incidents, one resulted in no harm to the animals, but failure to obtain some of the experimental data. The other two incidents resulted in failure to obtain some of the experimental data and made the animals unsuitable for the experiments. Although some of these animals had no pain and distress, they were euthanized because there was no

177 other experiment for which they were suitable.

\section{$178 \quad$ Novel classification system}

To resolve the complexity of the above results, we designed a novel ISCLA based on the degree of harm to the animals, the degree of harm to the experiments, and

181 the need to euthanize the animals (Table 1). According to the ISCLA, incidents not

182 resulting in harm (Category 0 and 1 ) constituted half of the total number of incidents ( $n$

$183=11,47.8 \%$; Fig. 3). Three (13.0\%) and six (26.1\%) incidents were classified into

184 Category 2 and 3a, respectively (Fig. 3). In addition, one incident, which harmed the experiment but not the animals, and two incidents, which resulted in euthanasia of the

186 animals, were classified into Category $3 \mathrm{~b}$ and 4a, respectively (Fig. 3). No incident was 187 classified into either Category 4 b or 5 (Fig. 3). 
Figure 4 shows the distribution of the incident severity by occurrence factors and work locations. Regarding the occurrence factor, the incidents associated with the equipment factor had a more severe impact than those associated with the human factor (Fig. 4A; $P=0.039$ ). All incidents associated with the equipment factor were classified

193 into Category 3a, whereas the more severe incidents classified into Category $3 \mathrm{~b}$ and $4 \mathrm{a}$ were associated with the human factor. One incident associated with the equipment factor occurred in the general area, whereas the other four occurred in the isolator area.

197 housing area, and seven (30.4\%) in the supporting area (Fig. 4B). The incidents

198 occurring in the animal housing area had a more severe impact on the animals and experiments than those of the supporting area (Fig. 4B; $P=0.002)$. Of the 16 incidents occurring in the animal housing area, nine occurred in the general area and seven in the isolator area (Fig. 4C). In addition, incidents occurring in the isolator area had more severe consequences than those in the general area (Fig. $4 \mathrm{C} ; P=0.001)$.

Discussion

We analyzed the incidents reported on our IRS that was conducted to take improvement measures of unexpected incidents. As a result, the number of incidents involving animal caretakers was the highest, and the occurrence of incidents was biased by day of the week. In addition, to evaluate the severity of the incident, it was necessary to comprehensively evaluate the degree of harm to the animals, the degree of harm to

210 the experiments, and the need to euthanize the animals. Based on the results, we

211 developed a novel ISCLA. To our knowledge, this is the first study to systematically

212 summarize the IRS conducted in a laboratory animal facility. 
214 statistically significant when weekends were excluded. At our facility, the work content

215 and the number of animal caretakers on weekends were lower than those on weekdays.

216 In addition, even on weekdays, the work content differed depending on the day of the

217 week. In the work of pharmacists, the major factor contributing to their dispensing

218 errors were high prescription volumes, pharmacist fatigue, and overworking [11].

219 Therefore, the bias of incident occurrences by day of the week might be affected by the

220 animal caretakers' work volume, work content, or fatigue, but the number of incidents

221 was too small to evaluate this in this study.

222 The incident severity classification developed in this study had one major

223 difference from similar classification systems used in the human medical field [9, 10,

224 12] in that incidents that had a large impact on the experiment that required euthanasia

225 of the animals were classified into Category $4 \mathrm{a}$, which has a relatively large impact even

226 without animal pain or distress. For instance, if animal results are mistaken in an

227 incorrect experimental procedure, which results in an unachievable experimental

228 purpose, the experiment is stopped and the animals are potentially euthanized,

229 regardless of their pain and distress. We considered that such incidents would have large

230 negative consequences on laboratory animal welfare from the perspective of

231 "Reduction" of the three Rs principle. In addition, although they were not included in

232 the incidents analyzed in this study, we noted incidents that cause considerable pain and

233 distress to animals and necessitate their euthanasia as Category $4 \mathrm{~b}$, and those that result

234 in death of the animals as Category 5. In total, the classification system that we

235 developed has eight categories. The 23 incidents were categorized without confusion

236 using the ISCLA. This suggested that each category in the ISCLA was properly defined. 

associated with the human factor. However, four-fifth of the incidents associated with the equipment factor occurred in the isolator area where more severe incidents occurred. In addition, the more severe incidents classified into category $3 b$ and $4 a$, which occurred 241 in the isolator area, were associated with the human factor. Therefore, the difference 242 between the human and equipment factors in terms of severity was not clarified in this study. When the incident cases accumulate, difference in severity between these factors would be clarified. Regarding the work locations, the more severe incidents in the isolator area suggested that incident detection tended to be delayed in isolators and that

246 the operational restriction in isolators caused difficulties in preventing the incidents.

247 Furthermore, categorizing incident severity using the ISCLA would aid in determining the priority of responses to the incidents. The ISCLA would also be available for evaluating the effectiveness of improvement measures based on not only the number of incident occurrences but also the degree of incident severity. These indicate that the ISCLA would be useful not only for taking improvement measures based on individual incidents but also for taking improvement measures from broader perspectives. The incidents analyzed in this study were reported on the IRS conducted at only one facility and in which the animal species housed were limited to mice and rats. In addition, investigators had not been encouraged to report in our IRS. This may have contributed to the low number of incidents analyzed that involved the investigators.

257 Therefore, evaluation of the appropriateness of the ISCLA at various laboratory animal facilities and in various IRSs is required. had characteristics that could not be seen from individual incidents alone. In addition, 
the ISCLA was considered useful when analyzing incidents and taking improvement measures in conducting the IRS in laboratory animal facilities.

\section{Acknowledgments}

The authors thank the animal caretakers and investigators for their cooperation with our incident reporting system.

\section{References}

1. Mohan S, Hampton LL, Silk SB. Adverse events at research facilities. Lab Anim (NY). 2017; 46: 244-249.

2. Koch VW. Preventing adverse events at research facilities. J Am Assoc Lab Anim Sci. 2018; 57: 660-669.

3. Garrouste-Orgeas M, Philippart F, Bruel C, Max A, Lau N, Misset B. Overview of medical errors and adverse events. Ann Intensive Care. 2012; 2: 2.

4. Hartnack S, Bettschart-Wolfensberger R, Driessen B, Pang

D, Wohlfender F. Critical incidence reporting systems - an option in equine anaesthesia? Results from a panel meeting. Vet Anaesth Analg. 2013; 40: e3-e8.

5. Wallis J, Fletcher D, Bentley A, Ludders J. Medical errors cause harm in veterinary hospitals. Front Vet Sci. 2019; 6:12.

6. Ludders JW, McMillan M. Erros in veterinary anesthesia. Iowa: Wiley Blackwell;

282 7. Bischoff S, Trietschel D, Enkelmann A, Schiffner R, Estrade P. Learning from negative results-Critical Incident Reporting System in Laboratory Animal Science 
285 8. Garfield S, Reynolds M, Dermont L, Franklin BD. Measuring the severity of 286 prescribing errors: a systematic review. Drug Saf. 2013; 36: 1151-1157.

287 9. Gates PJ, Baysari MT, Mumford V, Raban MZ, Westbrook JI. Standardizing the 288 classification of harm associated with medication errors: the harm associated with 289 medication error classification (HAMEC). Drug Saf. 2019; 42: 931-939.

290 10. [National University Hospital Council of Japan] [Internet]. Tokyo: National 291 University Hospital Council of Japan; c2019. [Incident severity classification]; [cited 2021 Jan 27]; $\left[\begin{array}{ll}1 & \text { p.]. Available from: }\end{array}\right.$ 293 http://nuhc.jp/Portals/0/images/activity/report/sgst_category/safety/incidentcategor $294 \quad$ y.pdf Japanese.

295 11. Peterson GM, Wu MS, Bergin JK. Pharmacist's attitudes towards dispensing errors: their causes and prevention. J Clin Pharm Ther. 1999; 24: 57-71.

297 12. Hartwig SC, Denger SD, Schneider PJ. Severity-indexed, incident report-based medication error-reporting program. Am J Hosp Pharm. 1991; 48: 2611-2616. 


\section{Figure Legends}

301 Figure 1. Occurrences and detections of incidents by time. (A) Occurrences of incidents

302 by month ( $n=23$ ). (B) Occurrences (black bar) and detections (white bar) of incidents

303 by day of the week ( $n=22$ and $n=23$, respectively). 1) Analysis including weekends,

304 2) Analysis excluding weekends.

305

306 Figure 2. Summary of harm to animals and experiments. Harm to animals was

307 classified by continuity as none, transient, permanent, and death, and severity as none,

308 mild, moderate, and severe. Harm to experiments was classified as none, possibility,

309 data missing, and not achieved. The incidents were also classified according to whether

310 they resulted in euthanasia of the animal. No incident resulting in permanent harm to

311 animals, death of an animal, or an unachieved experiment was reported.

312

313 Figure 3. Incidents by severity classification. Reported incidents were classified

314 according to the incident severity classification for laboratory animals (ISCLA) (Table

$3151)$.

316

317 Figure 4. Severity of the incidents by occurrence factor and work location. (A) The

318 incidents associated with the equipment factor (black diamond) exhibited more severe

319 outcomes than those associated with the human factor (white diamond). (B) The

320 incidents occurring in the animal housing area (black circle) had more severe outcomes

321 than those in the supporting area (white circle). (C) The incidents occurring in the

322 isolator area had more severe outcomes than those in the general area. 
A

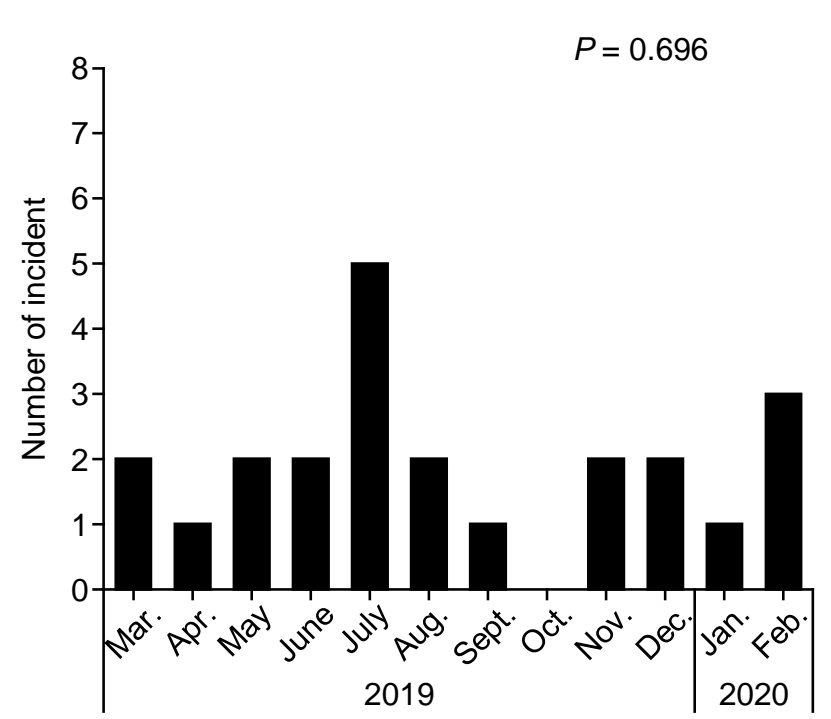

B

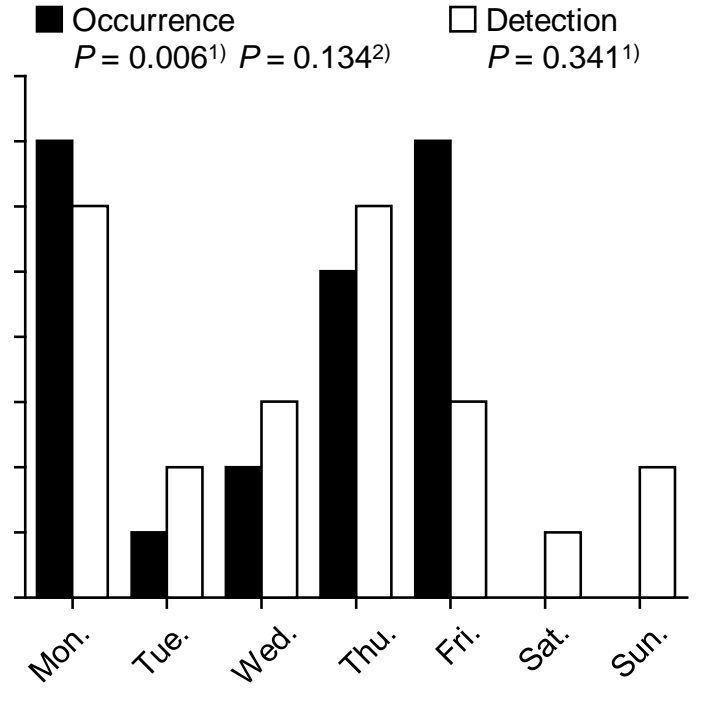

Figure 1. Occurrences and detections of incidents by time. 


\begin{tabular}{|c|c|c|c|c|}
\hline $\begin{array}{l}\text { Report } \\
\text { No. }\end{array}$ & \multicolumn{2}{|c|}{ Harm to animal } & $\begin{array}{c}\text { Harm to } \\
\text { experiment }\end{array}$ & Euthanasia \\
\hline 1 & Transient & Moderate & Data missing & Yes \\
\hline 2 & None & None & Data missing & Yes \\
\hline 3 & None & None & Data missing & No \\
\hline 4 & Transient & Moderate & Possibility & No \\
\hline 5 & Transient & Moderate & Possibility & No \\
\hline 6 & Transient & Moderate & None & No \\
\hline 7 & Transient & Moderate & None & No \\
\hline 8 & Transient & Moderate & None & No \\
\hline 9 & Transient & Moderate & None & No \\
\hline 10 & Transient & Mild & None & No \\
\hline 11 & Transient & Mild & None & No \\
\hline 12 & Transient & Mild & None & No \\
\hline 13 & None & None & None & No \\
\hline 14 & None & None & None & No \\
\hline 15 & None & None & None & No \\
\hline 16 & None & None & None & No \\
\hline 17 & None & None & None & No \\
\hline 18 & None & None & None & No \\
\hline $19-23$ & & Not affe & hear miss) & \\
\hline
\end{tabular}

Figure 2. Summary of harm to animals and experiments. 


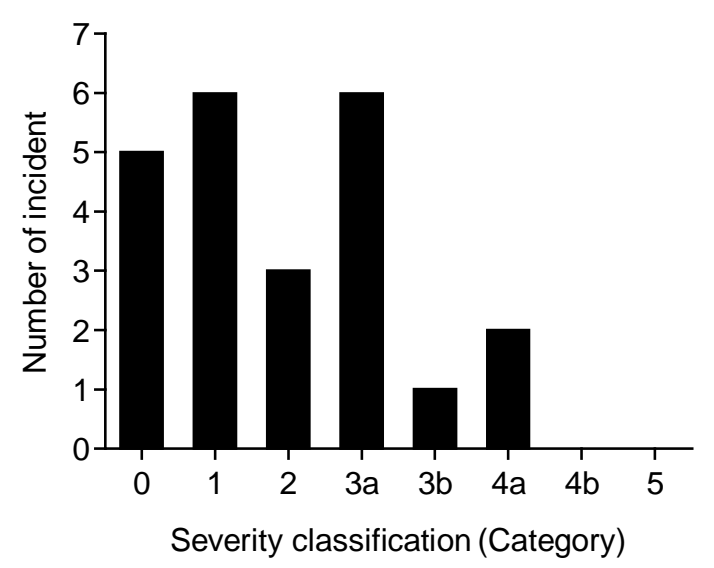

Figure 3. Incidents by severity classification. 

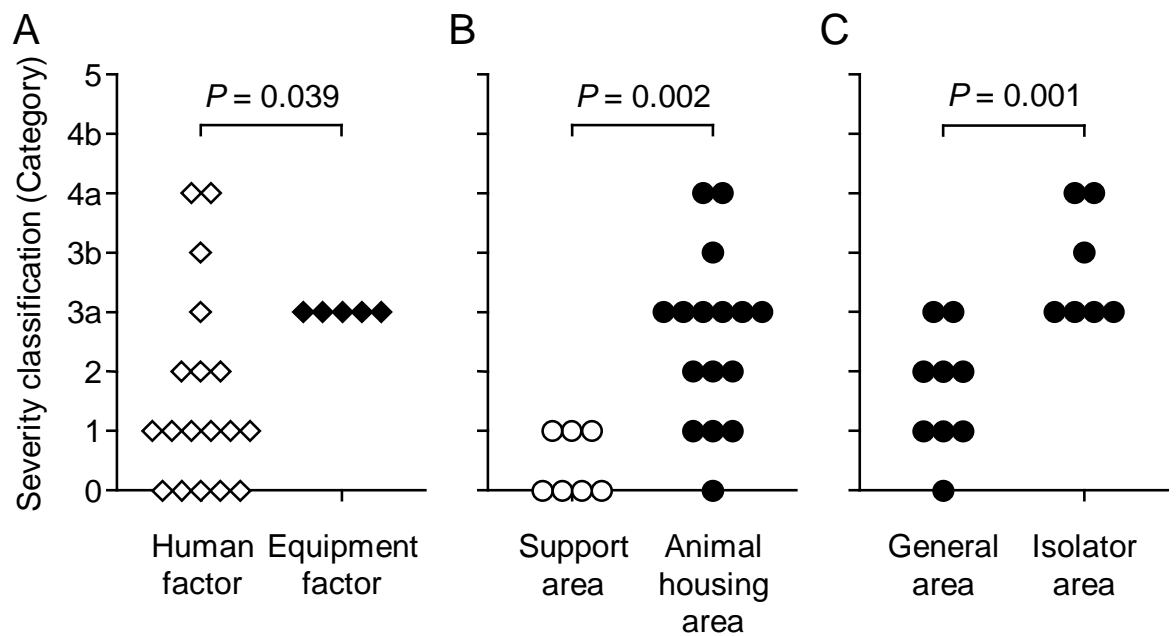

Figure 4. Severity of the incidents by occurrence factor and work location. 
Table 1 . Incident severity classification for laboratory animals (ISCLA)

\begin{tabular}{|c|c|c|c|c|c|}
\hline \multirow{2}{*}{ Category } & \multicolumn{2}{|c|}{ Influence on animals } & \multirow{2}{*}{$\begin{array}{l}\text { Influence on } \\
\text { experiment }\end{array}$} & \multirow{2}{*}{$\begin{array}{l}\text { Euthanasia } \\
\text { of animals }\end{array}$} & \multirow{2}{*}{ Outcome and treatment } \\
\hline & Continuity & Severity & & & \\
\hline 5 & Death & - & - & - & - Death \\
\hline $4 b$ & Permanent & Moderate-severe & Yes & Yes & $\begin{array}{l}\text { - Euthanization of the animals was required by permanent disability or } \\
\text { significant dysfunction } \\
\text { - Additional experiment was required by the influence on the experiment }\end{array}$ \\
\hline $4 a$ & - & - & Yes & Yes & $\begin{array}{l}\text { - Euthanization of the animals was required by deviation from the protocol or } \\
\text { difficulty in achieving the experimental purpose }\end{array}$ \\
\hline $3 b$ & Transient & Severe & Yes & $\mathrm{No} / \mathrm{Yes}$ & $\begin{array}{l}\text { - Substantial treatment for the animals was required } \\
\text { - Some experimental data were missing, but the experiment did not have to be } \\
\text { redone }\end{array}$ \\
\hline $3 a$ & Transient & Moderate & Yes & No & $\begin{array}{l}\text { - Simple treatment for the animals was required } \\
\text { - The purpose of the experiment was achieved, but the experiment might have } \\
\text { been somewhat influenced }\end{array}$ \\
\hline 2 & Transient & Mild & No & No & - Treatment for the animals was not necessary \\
\hline 1 & None & - & No & No & $\begin{array}{l}\text { - No animal was harmed, but the animal and experiment might have been } \\
\text { influenced }\end{array}$ \\
\hline 0 & - & - & - & - & - Error or trouble was found, but did not affect the animals \\
\hline
\end{tabular}

